\title{
On a theorem of Erdős, Rubin, and Taylor on choosability of complete bipartite graphs
}

\author{
Alexandr Kostochka * \\ University of Illinois at Urbana-Champaign, Urbana, IL 61801 \\ and Institute of Mathematics, Novosibirsk 630090, Russia \\ kostochk@math.uiuc.edu \\ Submitted: April 10, 2002; Accepted: August 13, 2002. \\ MR Subject Classifications: 05C15, 05C65
}

\begin{abstract}
Erdös, Rubin, and Taylor found a nice correspondence between the minimum order of a complete bipartite graph that is not $r$-choosable and the minimum number of edges in an $r$-uniform hypergraph that is not 2-colorable (in the ordinary sense). In this note we use their ideas to derive similar correspondences for complete $k$ partite graphs and complete $k$-uniform $k$-partite hypergraphs.
\end{abstract}

\section{Introduction}

Let $m(r, k)$ denote the minimum number of edges in an $r$-uniform hypergraph with chromatic number greater than $k$ and $N(k, r)$ denote the minimum number of vertices in a $k$-partite graph with list chromatic number greater than $r$.

Erdös, Rubin, and Taylor [6, p. 129] proved the following correspondence between $m(r, 2)$ and $N(2, r)$.

Theorem 1 For every $r \geq 2, m(r, 2) \leq N(2, r) \leq 2 m(r, 2)$.

This nice result shows close relations between ordinary hypergraph 2-coloring and list coloring of complete bipartite graphs. Note that $m(r, 2)$ was studied in $[2,3,4,9,10]$. Using known bounds on $m(r, 2)$, Theorem 1 yields the corresponding bounds for $N(2, r)$ :

$$
c 2^{r} \sqrt{\frac{r}{\ln r}} \leq N(2, r) \leq C 2^{r} r^{2} .
$$

*This work was partially supported by the NSF grant DMS-0099608 and the Dutch-Russian Grant NWO-047-008-006. 
Theorem 1 can be extended in a natural way in two directions: to complete $k$-partite graphs and to $k$-uniform $k$-partite hypergraphs. In this note we present these extensions (using the ideas of Erdős, Rubin, and Taylor).

A vertex $t$-coloring of a hypergraph $H$ is panchromatic if each of the $t$ colors is used on every edge of $G$. Thus, an ordinary 2-coloring is panchromatic. Some results on the existence of panchromatic colorings for hypergraphs with few edges can be found in [8]. Let $p(r, k)$ denote the minimum number of edges in an $r$-uniform hypergraph not admitting any panchromatic $k$-coloring. Note that $p(r, 2)=m(r, 2)$. The first extension of Theorem 1 is the following.

Theorem 2 For every $r \geq 2$ and $k \geq 2, p(r, k) \leq N(k, r) \leq k p(r, k)$.

It follows from Alon's results in [1] that for some $c_{2}>c_{1}>0$ and every $r \geq 2$ and $k \geq 2$,

$$
\exp \left\{c_{1} r / k\right\} \leq N(k, r) \leq k \exp \left\{c_{2} r / k\right\} .
$$

Therefore, by Theorem 2 we get reasonable bounds on $p(r, k)$ for fixed $k$ and large $r$ :

$$
\exp \left\{c_{1} r / k\right\} / k \leq p(r, k) \leq k \exp \left\{c_{2} r / k\right\} .
$$

Note that the lower bound on $p(r, k)$ with $c_{1}=1 / 4$ follows also from Theorem 3 of the seminal paper [5] by Erdős and Lovász.

We say that a $k$-uniform hypergraph $G$ is $k$-partite, if $V(G)$ can be partitioned into $k$ sets so that every edge contains exactly one vertex from every part. Let $Q(k, r)$ denote the minimum number of vertices in a $k$-partite $k$-uniform hypergraph with list chromatic number greater than $r$. Note that $Q(2, r)=N(2, r)$.

Theorem 3 For every $r \geq 2$ and $k \geq 2, m(r, k) \leq Q(k, r) \leq k m(r, k)$.

From [4] and [7] we know that

$$
c_{1} k^{r}\left(\frac{r}{\ln r}\right)^{1-1 /\left\lfloor 1+\log _{2} k\right\rfloor} \leq m(r, k) \leq c_{2} k^{r} r^{2} \log k .
$$

Thus, Theorem 3 yields that

$$
c_{1} k^{r}\left(\frac{r}{\ln r}\right)^{1-1 /\left\lfloor 1+\log _{2} k\right\rfloor} \leq Q(k, r) \leq c_{2} k^{r+1} r^{2} \log k .
$$

\section{Proof of Theorem 2}

Let $H=(V, E)$ be an $r$-uniform hypergraph not admitting any panchromatic $k$-coloring with $E=\left\{e_{1}, \ldots, e_{p(r, k)}\right\}$. Consider the complete $k$-partite graph $G=(W, A)$ with parts $W_{1}, \ldots, W_{k}$ and $W_{i}=\left\{w_{i, 1}, \ldots, w_{i,|E|}\right\}$ for $i=1, \ldots, k$. The ground set for lists will be $V$. Recall that every $e_{i}$ is an $r$-subset of $V$. For every $i=1, \ldots, k$ and $j=1, \ldots,|E|$, assign to $w_{i, j}$ the list $L\left(w_{i, j}\right)=e_{j}$. 
Assume that $G$ has a coloring $f$ from the lists. Since $G$ is a complete $k$-partite graph, every color $v$ is used on at most one part. Then $f$ produces a $k$-coloring $g_{f}$ of $V$ as follows: we let $g_{f}(v)$ be equal to the index $i$ such that $v=f\left(w_{i, j}\right)$ for some $j$ or be equal to 1 if there is no such $w_{i, j}$ at all. Since for every $j$ all vertices in $\left\{w_{1, j}, w_{2, j}, \ldots, w_{k, j}\right\}$ must get different colors, $g_{f}$ is a panchromatic $k$-coloring of $H$, a contradiction. This proves that $N(k, r) \leq k p(r, k)$.

Now, consider a complete $k$-partite graph $G=(W, A)$ with parts $W_{1}, \ldots, W_{k}$ and $|W|<p(r, k)$. Let $L$ be an arbitrary $r$-uniform list assignment for $W$. Let $H=(V, E)$ be the hypergraph with $V=\bigcup_{w \in W} L(w)$ and $E=\{L(w) \mid w \in W\}$. Since $|E|=|W|<$ $p(r, k)$, there exists a panchromatic $k$-coloring $g$ of $H$. Define the coloring $f_{g}$ of $W$ as follows: if $w \in W_{i}$, choose in the edge $L(w)$ of $H$ any vertex $v$ with $g(v)=i$ and let $f_{g}(w)=v$. Then vertices in different $W_{i}$ cannot get the same color, and $f$ is a coloring from the lists of vertices in $G$. This proves that $N(k, r) \geq p(r, k)$.

\section{Proof of Theorem 3}

Let $H=(V, E)$ be an $r$-uniform hypergraph not admitting any $k$-coloring with $E=$ $\left\{e_{1}, \ldots, e_{m(r, k)}\right\}$. Consider the complete $k$-partite $k$-uniform hypergraph $G=(W, A)$ with parts $W_{1}, \ldots, W_{k}$ and $W_{i}=\left\{w_{i, 1}, \ldots, w_{i,|E|}\right\}$ for $i=1, \ldots, k$. The ground set for lists will be $V$. Recall that every $e_{i}$ is an $r$-subset of $V$. For every $i=1, \ldots, k$ and $j=1, \ldots,|E|$, assign $w_{i, j}$ the list $L\left(w_{i, j}\right)=e_{j}$.

Assume that $G$ has a coloring $f$ from the lists. Note that no color $v$ is present on every $W_{i}$, since otherwise $G$ would have an edge with all vertices of color $v$. Thus, $f$ produces

a $k$-coloring $g_{f}$ of $V$ as follows: we let $g_{f}(v)$ be equal to the smallest $i$ such that $v$ is not a color of any vertex in $W_{i}$. Assume that $g_{f}$ is not a proper coloring, i.e., that some $e_{j}$ is monochromatic of some color $i$ under $g_{f}$. But some $v^{\prime} \in e_{j}$ must be $f\left(w_{i, j}\right)$, and therefore $g_{f}\left(v^{\prime}\right) \neq i$, a contradiction. This proves that $Q(k, r) \leq k m(r, k)$.

Now, consider a complete $k$-partite $k$-uniform hypergraph $G=(W, A)$ with parts $W_{1}, \ldots, W_{k}$ and $|W|<Q(r, k)$. Let $L$ be an arbitrary $r$-uniform list for $W$. Let $H=$ $(V, E)$ be the hypergraph with $V=\bigcup_{w \in W} L(w)$ and $E=\{L(w) \mid w \in W\}$. Since $|E|=|W|<Q(r, k)$, there exists a $k$-coloring $g$ of $H$. Define the coloring $f_{g}$ of $W$ as follows: if $w \in W_{i}$, choose the next number $i^{\prime}$ after $i$ in the cyclic order $1,2, \ldots, k$ such that there is a vertex $v^{\prime} \in L(w)$ with $g\left(v^{\prime}\right)=i^{\prime}$ and let $f_{g}(w)=v^{\prime}$. Since $L(w)$ is not monochromatic in $g$, we have $i^{\prime} \neq i$. On the other hand, no $v$ with $g(v)=i^{\prime}$ will be used to color a $w \in W_{i^{\prime}}$. Thus $f_{g}$ is a proper coloring of $G$. This proves that $Q(k, r) \geq m(r, k)$.

Acknowledgment. I thank both referees for the helpful comments.

\section{References}

[1] N. Alon, Choice number of graphs: a probabilistic approach, Combinatorics, Probability and Computing, 1 (1992), 107-114. 
[2] J. Beck, On 3-chromatic hypergraphs, Discrete Math. 24 (1978), 127-137.

[3] P. Erdős, On a combinatorial problem, I, Nordisk. Mat. Tidskrift, 11 (1963), 5-10.

[4] P. Erdős, On a combinatorial problem, II, Acta Math. Hungar., 15 (1964), 445-447.

[5] P. Erdős, L. Lovász, Problems and Results on 3-chromatic hypergraphs and some related questions, In Infinite and Finite Sets, A. Hajnal et. al., editors, Colloq. Math. Soc. J. Bolyai, 11, North Holland, Amsterdam, 609-627, 1975.

[6] P. Erdős, A.L. Rubin and H. Taylor, Choosability in graphs, Proc. West Coast Conf. on Combinatorics, Graph Theory and Computing, Congressus Numerantium XXVI (1979), 125-157.

[7] A.V. Kostochka. Coloring uniform hypergraphs with few colors, submitted.

[8] A.V. Kostochka and D. R. Woodall, Density conditions for panchromatic colourings of hypergraphs, Combinatorica, 21 (2001), 515-541,

[9] J. Radhakrishnan and A. Srinivasan, Improved bounds and algorithms for hypergraph two-coloring, Random Structures and Algorithms, 16 ( 2000), 4-32.

[10] J. Spencer, Coloring $n$-sets red and blue, J. Comb.Theory Ser. A, 30 (1981), 112 113. 